\title{
Safety, immunogenicity and effectiveness of inactivated influenza vaccines in healthy pregnant women and children under 5 years: An Evidence-Based Clinical Review
}

\author{
Amit Bansal ${ }^{1}$, Mai-Chi Trieu ${ }^{1}$, and Rebecca Cox $^{1}$ \\ ${ }^{1}$ University of Bergen Faculty of Medicine and Dentistry
}

July 15, 2020

\begin{abstract}
Background: Pregnant women and young children are at high risk for influenza complications and, therefore, recommended for annual influenza vaccination. However, most studies investigating the safety, immunogenicity and effectiveness of inactivated influenza vaccines (IIV) were conducted in healthy adults. Therefore, the safety, immunogenicity and effectiveness of IIV in pregnant women and young children are underexplored. Objective: In this review, we evaluate the safety profile, immunogenicity, and effectiveness of IIV in healthy pregnant women and children $<5$ years old. Methods: We searched the electronic databases PubMed, Google Scholar, MEDLINE, Embase, WHO International Clinical Trials Registry Platform (ICTRP), and UpToDate up until 8th June 2020. Selection criteria included publications assessing safety, immunogenicity and effectiveness of IIV in healthy pregnant women and children $<5$ years. Results: A total of 60 studies were selected for the review: 9 on IIV safety, 17 and 11 on immunogenicity, and 13 and 10 on effectiveness of IIV in pregnant women and children, respectively. Most randomized controlled trials in pregnant women included in this review were conducted in low- and middle-income countries while observation studies were conducted in high-income countries. Conclusion: IIV were found to be a safe preventive strategy with moderate immunogenicity and effectiveness estimates for pregnant women and children $<5$ years old. However, the effect sizes depended upon the study design, individual factors, vaccine type and manufacturing practices, and the antigenic match between the influenza vaccine strains and the circulating strains.
\end{abstract}

\section{Hosted file}

Safety, immunogenicity and effectiveness of IIV in pregnant women and children under 5 years 210620. doc available at https://authorea.com/users/343025/articles/469738-safety-immunogenicity-andeffectiveness-of-inactivated-influenza-vaccines-in-healthy-pregnant-women-and-childrenunder-5-years-an-evidence-based-clinical-review 